\title{
Fotoféresis: nueva terapia inmunomoduladora para enfermedades mediadas por linfocitos $\mathrm{T}$
}

\author{
J. M. VAGACE VALERO, N. ALONSO ESCOBAR, D. DE ARGILA FERNÁNDEZ- \\ DURÁN ${ }^{1}$, L. VARGAS PÉREZ ${ }^{2}$, J. MELERO RUIZ ${ }^{2}$, J. M. MORÁN PENCO ${ }^{3}$, R. BAJO \\ GÓMEZ $^{4}$, M. PÉREZ MIRANDA ${ }^{4}$
}

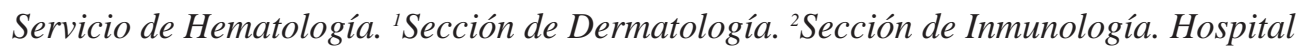
Infanta Cristina. ${ }^{3}$ Unidad de Experimentación Animal. ${ }^{4}$ Cátedra de Patología y Clínica Médica. Facultad de Medicina. Universidad de Extremadura. Badajoz.

\author{
PHOTOPHERESIS: NEW IMMUNOMODULATORY THERAPY FOR \\ T- LYMPHOCYTE MEDIATED DISEASES
}

\begin{abstract}
RESUMEN
La fotoféresis extracorpórea (FEC) es un procedimiento terapéutico basado en el efecto biológico del psoraleno y la luz ultravioleta A sobre las células mononucleares recogidas mediante aféresis y reinfundidas posteriormente al paciente. En 1988, este tratamiento fue la primera terapia inmunomoduladora aprobada por la FDA para cualquier tipo de cáncer. Datos convincentes recogidos en más de 160 centros en Europa y EE.UU. en estos años han documentado que el procedimiento tiene muy pocos efectos secundarios, prolonga la supervivencia e induce un 50 a $75 \%$ de respuestas en pacientes con linfoma T cutáneo avanzado. Además numerosos trabajos indican que la FEC es un potente agente para el tratamiento del rechazo del trasplante de órgano sólido, la enfermedad injerto contra huésped, la esclerodermia y otras enfermedades autoinmunes resistentes al tratamiento convencional. El efecto del tratamiento es debido probablemente a la inducción de una respuesta inmune celular anticlonotípica frente a las clonas de linfocitos T patológicos.
\end{abstract}

PALABRAS CLAVE: Fotoquimioterapia extracorpórea. Fotoféresis. Inmunomodulación.

\begin{abstract}
The photopheresis (ECP) is a therapeutic approach based on the biological effect of psoralen and ultraviolet light A on mononuclear cells collected by apheresis, and reinfused into the patient. In 1988, the treatment was the first FDA-approved selective immunotherapy for any type of cancer. Convincing data taken from over 160 centers in Europe and the U.S.A. over the past few years have documented that ECP is associated with a very low side-effect profile. Evidence shows that this therapy prolongs the mean survival, and also induces 50-75\% response rates in patients with advanced cutaneous T-cell lymphoma. In addition, more and more reports indicate that photopheresis is a potent agent in the therapy of solid organ transplant rejection, graft versus host disease, scleroderma, and other autoimmune diseases resistant to conventional therapy. The mechanism of this treatment is likely due to the induction of cell-mediated anticlonotypic immune response against pathogenic clones of T lymphocytes.
\end{abstract}

KEY WORDS: Extracorporeal Photochemotherapy. Photopheresis. Biological response modifiers.

Vagace Valero JM, Alonso Escobar N, Diego de Argila Fernández-Durán D, Vargas Pérez L, Melero Ruiz J, Morán Penco JM, Bajo Gómez $R$, Pérez Miranda M. Fotoféresis: nueva terapia inmunomoduladora para enfermedades mediadas por linfocitos T. An Med Interna (Madrid) 2003; $20: 421-426$

\section{INTRODUCCIÓN}

La fotoféresis, también llamada fotoquimioterapia extracorpórea (FEC) es una técnica de aféresis que consiste en la recolección de las células mononucleares de sangre periférica que son tratadas con psoraleno y radiación ultravioleta $\mathrm{A}$ y reinfundidas posteriormente al paciente. Se desarrolla así una respuesta inmune dirigida contra los linfocitos reinfundidos que es la base más probable del efecto terapéutico de la técnica (1).

La fotoféresis fue usada por primera vez por Edelson en el Linfoma T cutáneo (2). En la actualidad la técnica se aplica también a pacientes con rechazo de trasplantes de órgano, enfermedad injerto contra huésped o enfermedades autoinmunes refractarias al tratamiento inmunosupresor. La principal ventaja de este tratamiento es que no produce inmunodepresión y es muy bien tolerado clínicamente permitiendo suspender o disminuir las necesidades de inmunosupresores en estos pacientes (3).

\section{EL PROCEDIMIENTO DE FOTOFÉRESIS}

Brevemente la técnica es como sigue:

1. El paciente es sometido a una aféresis para obtener un concentrado de células mononucleares en un pequeño volumen $(<150 \mathrm{ml})$ en un tiempo aproximado de dos horas.

2. El concentrado linfo-monocitario es diluido hasta un volumen final de $300 \mathrm{cc}$ con salino y se le añade psoraleno (8MOP) soluble a una concentración de $200 \mathrm{ng} / \mathrm{ml}$. La mezcla

Financiación del trabajo: Proyecto de investigación financiado por el II Plan Regional de Investigación, Desarrollo Tecnológico e Innovación de Extremadura (2001-2004). Consejería de Educación Ciencia y Tecnología. Junta de Extremadura.

Trabajo aceptado: 24 de marzo de 2003

Correspondencia: José Manuel Vagace Valero. Apartado de Correos 667. Badajoz. e-mail: jvagacev@aehh.org. 


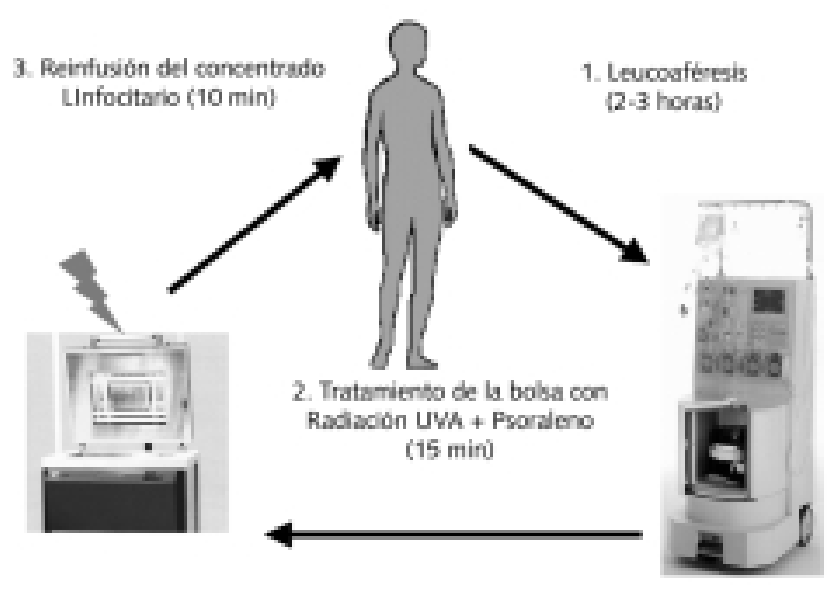

Fig. 1. El procedimiento de fotoféresis en tres tiempos.

se transfiere a una bolsa permeable a la radiación UVA y se somete a radiación UVA a una dosis total de $2 \mathrm{Julios} / \mathrm{cm}^{2}$ en un tiempo aproximado de $15 \mathrm{~min}$.

Posteriormente se infunde el concentrado al paciente con un equipo de transfusión

El procedimiento se repite 2 días consecutivos cada 2 ó 4 semanas según la patología a tratar (4).

- Coste del procedimiento: El coste estimado de cada proceso se calcula en unos $350 €$.

-Tolerancia clínica: La experiencia disponible en la actualidad en más de 150 centros con más de 200.000 procedimientos realizados permite asegurar que la fotoféresis no produce citopenias, no produce inmunosupresión y a las concentraciones utilizadas el 8-MOP, no es mutagénico. No se han comunicado hasta la fecha efectos secundarios severos ni episodios infecciosos relacionados con este tratamiento (5). Sólo se observó cefalea, escalofríos o fiebre relacionada con la infusión en menos del 4\% de los casos (6).

-Indicaciones de la fotoféresis (Tabla I): La fotoféresis es una indicación de primera línea en las formas eritrodérmicas del linfoma T cutáneo, en el resto de las indicaciones la FEC se considera hoy en día una terapia de segunda línea aplicable a aquellos pacientes refractarios o que no toleren el tratamiento inmunosupresor.

-Contraindicaciones de la fotoféresis (Tabla II): La alergia al psoraleno se considera la principal contraindicación para la FEC.

\section{TABLA I}

\section{ENFERM EDADES TRATADAS CO N FO TO FÉRESIS}

Linfoma T cutáneo

Enfermedades autoinmunes

Prevención y/o tratamiento del rechazo en el trasplante de órgano sólido

Prevención y/o tratamiento de la enfermedad injerto contra huésped

M iscelánea
TABLA ॥

CONTRAINDICACIONES DE LA FOTO FÉRESIS

Alergia al psoraleno

Insuficiencia cardíaca inestable o tratamiento reciente con IECA

Sepsis

Hipotensión sistólica

Linfopenia $<1000 / \mathrm{mm}^{3}$

Acceso venoso inadecuado

MECANISMO DE ACCIÓN DE LA FOTOFÉRESIS

¿CÓMO ACTÚA EL PSORALENO? (Fig. 2)

Los psoralenos son furocumarinas inertes que cuando se exponen a la radiación ultravioleta-A se activan durante milisegundos formando enlaces covalentes entre dos bases pirimidínicas, intercalándose entre las hebras del DNA e impidiendo su síntesis. Los linfocitos auto-reactivos y tumorales son más sensibles al efecto PUVA, quizás por presentar un mayor número de receptores para el psoraleno (7). En la membrana celular el fármaco puede modificar también otras macromoléculas. La consecuencia es que el linfocito tratado es más antigénico, sufre inhibición funcional completa y muere por apoptosis 48 o 72 horas después.

El monocito es resistente al efecto del psoraleno y sufre activación tras su paso por el circuito extracorpóreo transformándose en célula dendrítica (8).

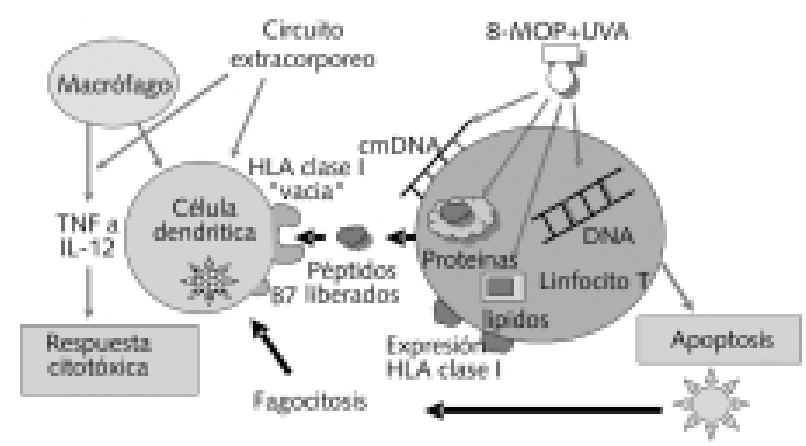

Fig. 2. Efecto de la técnica sobre el linfocito T y sobre el macrófago: sobre el linfocito, el psoraleno activado por UVA interacciona con la cadena de DNA nuclear, DNA de membrana, con las proteinas y con los lípidos, produciendo la muerte celular por apoptosis y aumentando la antigenicidad mediante el incremento en la expresión de antígenos de histocompatibilidad clase I y mediante la liberación de peptidos especificos. El monocito, más resistente a la acción del psoraleno, se activa en el circuito extracorpóreo, libera interleukinas que favorecen la respuesta T citotóxica y se trasforma en célula dendrítica capaz de captar los péptidos liberados por el linfocito T patológico.

\section{¿QUÉ OCURRE AL REINFUNDIR EL CONCENTRADO LINFOCITARIO TRATADO AL PACIENTE? (Fig. 3)}

Puesto que sólo se tratan en cada proceso menos del $5 \%$ de los linfocitos del organismo, el efecto del tratamiento sobre el resto se atribuyó a autoinmunidad inducida frente a los linfo- 


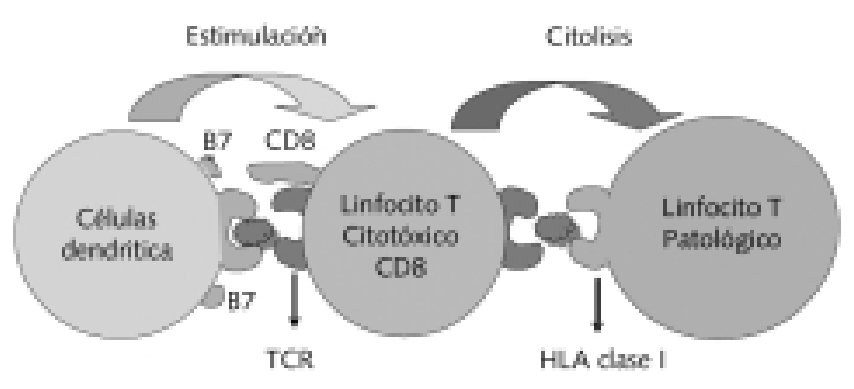

Fig. 3. Desarrollo de la respuesta inmune clonoespecífica: la figura representa como, una vez reinfundidos en la circulación, las células dendríticas, a través de moléculas coestimuladoras (B7), inducen la proliferación de células T8 citotóxicas dirigidas contra los antígenos linfocitarios que trasportan unidos a moléculas HLA clase I. Estas células citotóxicas podrán reconocer a otros linfocitos T patológicos y desencadenar una respuesta citotóxica clonoespecífica.

citos T patológicos, lo que se ha denominado teoría de la autovacunación T (9).

Numerosos modelos experimentales demuestran que el tratamiento induce una respuesta $\mathrm{T}$ citotóxica clonoespecífica contra los linfocitos $\mathrm{T}$ responsables de patología autoinmune o aloinmune. Los animales tratados conservan íntegra su respuesta y su memoria inmune (10).

\section{APLICACIONES CLÍNICAS DE LA FOTOFÉRESIS}

\section{LINFOMA T CUTÁNEO}

El linfoma $\mathrm{T}$ cutáneo fue la primera enfermedad tratada con FEC y es la única indicación aprobada por la FDA en la actualidad para este tratamiento.

El estudio prospectivo y multicéntrico realizado por Edelson y cols. (2) sobre 37 pacientes con linfoma T cutáneo resistentes al tratamiento, obtuvo una respuesta objetiva en 27 pacientes $(71 \%)$ con una disminución promedio del $60 \%$ en la afectación cutánea. Nueve pacientes entraron en remisión completa de los cuales 4 han permanecido libres de enfermedad sin tratamiento durante 10 años. El tratamiento fue muy bien tolerado y no se observó ninguno de los efectos secundarios asociados a la quimioterapia. El seguimiento a largo plazo de los pacientes de esta serie ha demostrado una supervivencia media de 60,3 meses desde el diagnóstico, superior a los controles históricos (11). Otros autores han encontrado supervivencias entre 90 y 100 meses $(12,13)$. Desde el trabajo de Edelson la experiencia comunicada con este tratamiento en numerosas publicaciones confirma los resultados iniciales, aunque con variabilidad en el porcentaje de respuestas que oscilan entre 50 y $75 \%$, con respuestas completas entre 15 y el $25 \%$ de los pacientes (14). Hoy se considera la única monoterapia que ha prolongado la supervivencia y es de primera indicación en pacientes con eritrodermia (15). La asociación con otros agentes inmunomoduladores puede mejorar la supervivencia de los pacientes con peor pronóstico (16).

La experiencia con más de 270 pacientes tratados en el mundo permite anticipar el tipo de paciente candidato a tener una buena respuesta a la fotoféresis (14) (Tabla III).
TABLA III

FACTORES ASO CIADOS A UNA BUENA RESPUESTA A LA FOTO FÉRESIS EN EL LINFOMA T CUTÁNEO

Forma eritrodérmica

Presencia del clon patológico en SP

Corta duración de la enfermedad

Número normal de linfocitos T8

Actividad NK conservada

No historia previa de quimioterapia

\section{ENFERMEDADES REUMATOLÓGICAS Y AUTOINMUNES}

-Esclerodermia: El primer estudio prospectivo, randomizado, multicéntrico y a simple ciego sobre 79 pacientes con esclerodermia de reciente inicio y con evidencia de progresión cutánea comparaba la FEC con la D-penicilamina (17). Evaluando biopsias y varios parámetros objetivos de afectación cutánea y pulmonar, la FEC obtuvo más respuestas (68 vs $32 \%$ a los 6 meses de tratamiento) y fue mejor tolerada por los pacientes. Desde entonces se han tratado más de 150 pacientes con esta técnica. Aunque algunos trabajos no encuentran beneficio objetivo (18), las respuestas comunicadas suponen un $45 \%$ del total de pacientes y se dan fundamentalmente sobre la rigidez cutánea y articular. Responden mejor las formas con progresión cutánea de inicio reciente ( $<$ de 2 años) (19) y aquellos pacientes que presentan en sangre periférica una población T clonal (20).

-Lupus eritematoso sistémico (LES): En 1992 (21) se trataron con fotoféresis mensual durante 6 meses y bimensual durante otros 6 , diez pacientes con formas leves o moderadas de la enfermedad. En siete casos se objetivó mejoría y en cinco casos la remisión de todas las manifestaciones clínicas que persistió hasta 30 meses después. Este tratamiento permitió disminuir drásticamente o suspender los fármacos inmunosupresores, así como el consumo de antiinflamatorios. Pese a los buenos resultados de este estudio no se ha realizado ningún otro ensayo clínico en el LES y sólo se ha comunicado algún caso aislado con buena evolución (22),

-Artritis reumatoide (AR): Malawista y cols., (23) obtuvieron respuestas objetivas en 4 de 7 pacientes tratados con fotoféresis en los cuales disminuyó el número y grado de afectación articular, los índices de dolor, la rigidez matutina y el grado de actividad global de la enfermedad. Como en el caso del LES, no se modificaron los parámetros analíticos de actividad. La respuesta se produjo tras seis semanas de tratamiento lo que en opinión de los autores es un dato en contra del posible efecto placebo. Otros 23 pacientes han sido tratados con respuesta favorable (24).

-Artritis psoriásica: En 8 pacientes tratados con FEC durante 24 semanas y evaluados cada $3 \mathrm{~m}$ hasta 1 año después de finalizado el tratamiento, 4 pacientes respondieron con un $74 \%$ de disminución en el índice de afectación articular de Ritchie ( $\mathrm{p}<0,01)$. Como en los casos anteriores, los parámetros de laboratorio se modificaron escasamente en estos pacientes (25).

-Pénfigo: En esta enfermedad autoinmune se han publicado varias series cortas de pacientes refractarios a corticoides 
e inmunosupresores que han logrado remisiones en la mayoría de los casos con fotoféresis (26) aunque con frecuencia los pacientes recidivan al espaciar las sesiones. Otras enfermedades dermatológicas como la dermatitis atópica severa o el Liquen plano han sido tratadas con éxito en casos refractarios al tratamiento esteroideo o inmunosupresor (27).

Una reciente revisión sobre la fotoféresis en enfermedades autoinmunes concluye que los sesgos de publicación, el carácter fluctuante de alguna de estas enfermedades y la dificultad para objetivar la respuesta en las mismas hacen difícil establecer el posible beneficio de este tratamiento siendo necesarios más ensayos clínicos controlados (28).

\section{RECHAZO DEL TRASPLANTE DE ÓRGANOS}

Diversos modelos experimentales (10) han demostrado que la fotoféresis puede controlar las manifestaciones del rechazo del trasplante sin producir inmunosupresión, generando una respuesta inmune contra las clonas de linfocitos T responsables del mismo. Constanzo-Nordin y cols. (29), en un estudio randomizado publicado en 1992 demostraron que una sola sesión de FEC resultaba equivalente a tres días de tratamiento con esteroides a dosis altas en el control del rechazo agudo del trasplante cardíaco. Los ensayos clínicos randomizados desarrollados en los últimos años, han demostrado que la FEC ha disminuido los episodios de rechazo agudo (30) y también las manifestaciones del rechazo crónico en el trasplante cardíaco (31).

La pauta de tratamiento son dos FEC semanales durante 15 días y posteriormente quincenales durante 2 ó 3 meses. El tratamiento inmunosupresor se mantiene inicialmente y posteriormente se va retirando según la evolución.

Una reciente revisión (32) resume los resultados de la fotoféresis en los trasplantes de órganos:

En el trasplante pulmonar se han publicado pequeñas series de pacientes en las cuales se ha mostrado eficaz en la bronquiolitis obliterante consecuencia del rechazo crónico.

En el trasplante renal, con la excepción de un trabajo que no obtuvo respuesta en tres pacientes tratados con pauta mensual, los demás trabajos publicados utilizando protocolos más agresivos obtienen buenos resultados en pequeñas series de pacientes con rechazo agudo y crónico resistente a los corticoides e inmunosupresores.

En resumen con una experiencia de 160 trasplantes tratados en el mundo la fotoféresis ha permitido tratar y prevenir con éxito el rechazo del trasplante y ofrece una alternativa terapéutica o profiláctica para aquellos pacientes con más riesgo de rechazo o en los cuales el tratamiento inmunosupresor esté contraindicado o resulte ineficaz (32).

\section{ENFERMEDAD INJERTO CONTRA HUÉSPED}

La enfermedad injerto contra huésped (EICH) es una complicación frecuente del trasplante alogénico de médula ósea o de precursores hematopoyéticos de sangre periférica, que se debe a la presencia de linfocitos $\mathrm{T}$ del donante que reaccionan frente a los antígenos de histocompatibilidad del receptor. Los órganos diana son el sistema inmunológico, la piel, el hígado y el intestino (33). Varios estudios han demostrado que la fotoféresis normaliza el cociente T4/T8, aumenta la población de células NK, induce apoptosis de los linfocitos T patogéni$\cos$ y disminuye su aloreactividad en estos pacientes (34).

Hasta la fecha no se han publicado estudios randomizados, pero sí numerosas series de pacientes tratados con FEC, tanto adultos, (35) como niños con formas refractarias al tratamiento inmunosupresor (36). La pauta de tratamiento es similar a la mencionada en el trasplante de órganos. Una reciente publicación resume los resultados comunicados hasta la fecha en un total de 76 pacientes con EICH aguda y 204 pacientes con EICH crónica. La fotoféresis consigue una mejoría muy significativa en la calidad de vida de los pacientes permitiendo disminuir o retirar el tratamiento inmunosupresor en la mayoría de ellos. En la EICH crónica remiten bien las alteraciones cutáneas $(76 \%)$, las alteraciones de la mucosa oral $(63 \%)$, la colestasis hepática (48\%) y la afectación pulmonar (39\%). En la EICH aguda remiten por completo las manifestaciones cutáneas en el 67\%, las manifestaciones hepáticas en el 38\% y las gastrointestinales en el 54\% de los casos (37). En esta enfermedad se aconseja iniciar la FEC si no hay respuesta después de 7 días con esteroides a $2 \mathrm{mg} / \mathrm{kg} /$ día (35).

\section{NUEVAS APLICACIONES CLÍNICAS DE LA FOTOFÉRESIS}

- Prevención de la reestenosis tras angioplastia coronaria transluminal (38). Se ha demostrado que en la reestenosis, además de material trombótico, hay un infiltrado de linfocitos $\mathrm{T}$, macrófagos y músculo liso que migran por el efecto de citoquinas. Se sabe que los factores autoinmunes juegan un importante papel en esta proliferación celular, con esta base se evaluó la fotoféresis en un estudio prospectivo, randomizado y controlado sobre 78 pacientes que sufrieron angioplastia de un sólo vaso. A los 6 meses ocurrió reestenosis en el 27\% del grupo control (41 pacientes) y sólo en el $8 \%$ del grupo tratado con 5 sesiones de FEC (37 pacientes).

-Enfermedad de Crohn (39). En un reciente estudio prospectivo se trataron 10 pacientes con enfermedad de Crohn corticodependientes mediante dos fotoréresis quincenales durante 24 semanas, 4 de ellos pudieron suspender los corticoides y otros 4 reducirlos al menos un 50\%. Pese a la reducción de los esteroides, en este grupo disminuyó la actividad de la enfermedad y los niveles de proteína $\mathrm{C}$ reactiva. Mejoró también la permeabilidad intestinal y la calidad de vida. En estos pacientes se detectaron linfocitos marcados con MOP en la mucosa intestinal.

- Hepatitis C resistente al IFN (40). La técnica obtiene un $20 \%$ de respuestas asociada al interferón en un estudio randomizado sobre 15 pacientes cirróticos infectados por el genotipo-1 y previamente resistentes al interferón. Aunque estas respuestas no son mantenidas, en la actualidad se está llevando a cabo un ensayo multicéntrico para evaluarlo.

-Esclerosis en placas (41). En el modelo experimental de esta enfermedad, la encefalomielitis alérgica del ratón, la fotoféresis se mostró eficaz. Con esta base los primeros pacientes tratados mostraron resultados alentadores con disminución sensible de las recaídas en un seguimiento de varios años respecto a las que habían presentado previamente, pero un reciente trabajo randomizado y a doble ciego no ha encontrado diferencias con el grupo placebo en la evolución de 16 pacientes estudiados. El carácter fluctuante de la enfermedad hace difícil sacar conclusiones definitivas con tan pocos pacientes con solo un año de seguimiento. 
- Diabetes tipo I de reciente inicio (42). Al tratarse de una enfermedad autoinmune de curso homogéneo, de fácil diagnóstico y seguimiento se convierte en un modelo muy adecuado para evaluar la utilidad de esta técnica inmunomoduladora. Un estudio sueco randomizado y a doble ciego (usando un simulacro de aféresis en el grupo control) sobre 49 niños con diabetes tipo I de reciente inicio demostró en el grupo tratado con FEC menos necesidades de insulina a igual tasa de $\mathrm{Hb}$ glicosilada comparado con el grupo control. Es de señalar que el tratamiento duró sólo 3 meses y el efecto seguía detectándose a los 3 años de la randomización, pese a que el grupo tratado tenía una diabetes más severa que el grupo control.

\section{CONCLUSIÓN}

La FEC es una terapia inmunomoduladora que se ha mostrado eficaz en diversas enfermedades mediadas por linfocitos $\mathrm{T}$ y se caracteriza por su escasa toxicidad comparada con la mayoría de los inmunosupresores. En la actualidad parece haber suficiente evidencia clínica de su utilidad en la forma eritrodérmica del linfoma $\mathrm{T}$ cutáneo, en la enfermedad injerto contra huésped y en el rechazo del trasplante de órgano sólido, pero existe un consenso generalizado sobre la necesidad de iniciar ensayos clínicos aleatorizados para precisar el papel que pueda tener este tratamiento en otras enfermedades autoinmunes mencionadas en esta revisión.

El mecanismo por el cual la FEC puede inducir respuestas clínicas dramáticas sigue siendo objeto de investigación. La apoptosis de los linfocitos $\mathrm{T}$ y la activación de las células presentadoras de antígeno induciendo una respuesta inmune clonoespecífica parece la hipótesis más probable. Es posible que en el futuro algunas modificaciones de la técnica como la expansión in vitro de los clones patológicos o su inmunoselección previa al tratamiento, la prolongación del tiempo de incubación o la adición de citoquinas al concentrado mononuclear puedan mejorar los resultados terapéuticos y probablemente serán la base para inmunoterapias más específicas y eficaces (43).

\section{Bibliografía}

1. Oliven A, Shechter Y. Extracorporeal photopheresis: a review. Blood Rev 2001; 15: 103-8.

2. Edelson R. Treatment of cutaneous T-Cell Lymphoma by extracorporeal photochemotherapy. N Engl J Med 1987; 316: 297-303.

3. Knobler R. Extracorporeal photochemotherapy-present and future-.Vox Sang 2000; 78 (Supl. 2): 197-201.

4. Schooneman F.Extracorporeal photopheresis technical aspects. Transfus Apheresis Sci 2003; 28: 51-61

5. Perotti C, Torretta L, Viarengo G, et al. Feasibility and safety of a new technique of extracorporeal photochemotherapy: experience of 240 procedures. Haematologica 1999; 84: 237-41.

6. Salvaneschi L, Perotti C, Torretta L. Adverse effects associated with extracorporeal photochemotherapy. Transfusion 2000; 40: 121.

7. Rook AH, Gottlieb SL, Wolfe JT, Vowels BR, Sood SS, Niu Z, et al. Pathogenesis of cutaneous T-cell lymphoma: implications for the use of recombinant cytokines and photopheresis. Clin Exp Immunol 1997; (Supl. 1):16-20

8. Tambur AR, Ortegel JW, Morales A, et al. Extracorporeal photopheresis induces lymphocyte but not monocyte apoptosis. Transplant Proc 2000; 32: 747-8.

9. Russell-Jones R. Shedding light in photopheresis. Lancet 2000; 17; 357: 32-3.

10. Van Iperen HP, Beijersbergen van Henegouwen GM, et al. Clinical and mechanistic aspects of photopheresis. J Photochem Photobiol B 1997; 39: 99-109.

11. Heald P, Rook A, Perez M, Wintroub B, Knobler R, et al. Treatment of erythrodermic cutaneous T-cell lymphoma with extracorporeal photochemotherapy. J Am Acad Dermatol 1992; 27: 427-33.

12. Zic JA, Stricklin GP, Greer JP, Kinney MC, Shyr Y, Wilson DC, et al. Long-term follow-up of patients with cutaneous T-cell lymphoma treated with extracorporeal photochemotherapy. J Am Acad Dermatol 1996; 35: 935-45.

13. Gottlieb SL, Wolfe JT, Fox FE, De Nardo BJ, Macey WH, Bromley PG, et al. Treatment of cutaneous T-cell lymphoma with extracorporeal photopheresis monotherapy and in combination with recombinant interferon alfa: a 10-year experience at a single institution. J Am Acad Dermatol 1996; 35: 946-57.

14. Knobler R, Jantschitsch C. Extracorporeal photochemoimmunotherapy in cutaneous T-cell lymphoma. Transfus Apheresis Sci 2003; 28 (1): $81-9$

15. Apisarnthanarax N, Talpur R and Duvic Madeleine. Treatment of Cutaneous T Cell Lymphoma. Current Status and Future Directions. Am J Clin Dermatol 2002; 3: 193-218.
16. Suchin KR, Cucchiara AJ, Gottleib SL, et al. Treatment of cutaneous T-cell lymphoma with combined immunomodulatory therapy: a 14year experience at a single institution. Arch Dermatol. 2002; 138: 1054-60.

17. Rook AH, Freundlich B, Jegasothy BV, Pérez MI, et al. Treatment of systemic sclerosis with extracorporeal photochemotherapy. Results of a multicenter trial. Arch Dermatol 1992; 128: 337-46.

18. Enomoto DN, Mekkes JR, Bossuyt PM, Yong SL. Treatment of patients with systemic sclerosis with extracorporeal photochemotherapy (photopheresis). J Am Acad Dermatol 1999; 41: 915-22.

19. Krasagakis K, Dippel E, Ramaker J, Owsianowski M, Orfanos CE. Management of severe scleroderma with long-term extracorporeal photopheresis. Dermatology 1998; 196: 309-15.

20. French LE, Lessin SR, Addya K. Identification of clonal T cells in the blood of patients with systemic sclerosis: positive correlation with response to photopheresis. Arch Dermatol 2001; 137: 1309-13.

21. Knobler RM, Graninger W, Graninger W, et al Extracorporeal photochemotherapy for the treatment of systemic lupus erythematosus. A pilot study. Arthritis Rheum 1992; 35: 319-24.

22. Lichtmbalyohere A, et al. Extracorporeal photochemotherapy of therapy-refractory cases of systemic lupus erythematosus with urticarial vasculitis and pemphigus foliaccus. Eur J Dermatol 1996; 6: 106-9.

23. Malawista SE, Trock DH, Edelson RL. Treatment of rheumatoid arthritis by extracorporeal photochemotherapy. A pilot study. Arthritis Rheum 1991; 34: 646-54.

24. Zic JA, Miller JL, Stricklin GP, King LE Jr. The North American experience with photopheresis. Ther Apher 1999; 3: 50-62.

25. Vahlquist C, Larsson M. Treatment of psoriatic arthritis with extracorporeal photochemotherapy and conventional psoralen-ultraviolet A irradiation. Arthritis Rheum 1996; 39 (9): 1519-23.

26. Wollina U, Lange D, Looks A. Short-time extracorporeal photochemotherapy in the treatment of drug-resistant autoimmune bullous diseases. Dermatology 1999; 198: 140-4.

27. Russo GG, Mullen C. Cutaneous and noncutaneous disorders treated with extracorporeal photopheresis. Int J Dermatol 2001; 40: 89-100.

28. Mayes MD. Photopheresis and autoimmune diseases. Rheum Dis Clin North Am 2000; 26: 75-81.

29. Costanzo-Nordin MR, Hubbell EA, et al. Photopheresis versus corticosteroids in the therapy of heart transplant rejection. Preliminary clinical report. Circulation 1992; 86 (Supl. 5): II242-50.

30. Barr ML, Meiser BM, Eisen HJ, et al. Photopheresis for the prevention of rejection in cardiac transplantation. Photopheresis Transplantation Study Group. N Engl J Med 1998; 339: 1744-51. 
31. Barr ML, Baker CJ, Schenkel FA, et al. Prophylactic photopheresis and chronic rejection: effects on graft intimal hyperplasia in cardiac transplantation. Clin Transplant 2000; 14: 162-6.

32. Dall'Amico R, Murer L. Extracorporeal photochemotherapy: a new therapeutic approach for allograft rejection. Transfus Apheresis Sci 2002; 26: 197-204.

33. James LM. Ferrara. Graft-Versus-Host Disease and Graft-versus-Leukemia Effect. En: Hoffman, et al. eds. Hematology: Basic Principles and Practice. Third edition. Churchill Livingstone, 2000: 1672-94.

34. Gorgun G, Miller KB, Foss FM. Immunologic mechanisms of extracorporeal photochemotherapy in chronic graft-versus-host disease. Blood 2002; 100: 941-7.

35. Greinix HT, Volc-Platzer B, Kalhs P, et al. Extracorporeal photochemotherapy in the treatment of severe steroid-refractory acute graft-versus-host disease: a pilot study. Blood 2000; 96: 2426-31.

36. Salvaneschi L, Perotti C, Zecca M, et al. Extracorporeal photochemotherapy for treatment of acute and chronic GVHD in childhood. Transfusion 2001; 41: 1299-305.

37. Dall'Amico R, Messina C. Extracorporeal photochemotherapy for the treat- ment of graft-versus-host disease. Ther Apher 2002; 6: 296-304.

38. Bisaccia E, Klainer AS, et al. Feasiblility of photophesis to reduce the occurrence of restenosis after percutaneous transluminal coronary angioplasty: A clinical pilot study. Am Heart J 2001; 142: 461-5.

39. Reinisch W, Nahavandi H, Santella R, et al. Extracorporeal photochemotherapy in patients with steroid-dependent Crohn's disease: a prospective pilot study. Aliment Pharmacol Ther 2001; 15: 1313-22.

40. O’Brien CB, Henzel BS, Monka DK, et al. Extracorporeal photopheresis along with interferón-alfa $2 \mathrm{a}$ in chronic hepatitis $\mathrm{C}$ patients who failled previous interferón therapy. Digest Dis Sci 1999; 44: 1020-6.

41. Rostami A, Sater R, Bird S, et al. A double blind placebo controlled trial of extracorporeal photopheresis in chronic progressive multiple sclerosis. Multiple Sclerosis 1999; 5: 198-203.

42. Ludvigsson J, Samuelsson U, Ernerudh J, et al. Photopheresis at onset of type 1 diabetes: a randomised, double-blind, placebo controlled trial. Arch Dis Child 2001; 85: 149-54.

43. Girardi M, Schechner J, Glusac E, Berger C, Edelson R. Transimmunization and the evolution of extracorporeal photochemotherapy. Transfus Apheresis Sci 2002; 26: 181-90. 